\title{
Virulence Profile and Genetic Structure of a North Dakota Population of Pyrenophora teres f. teres, the Causal Agent of Net Form Net Blotch of Barley
}

\author{
Z. H. Liu, S. Zhong, A. K. Stasko, M. C. Edwards, and T. L. Friesen
}

First, second, and fifth authors: Department of Plant Pathology, North Dakota State University, Fargo, ND 58105; and third, fourth, and fifth authors: Northern Crop Science Laboratory, United States Department of Agriculture-Agricultural Research Service, Fargo, ND 58102. Accepted for publication 30 January 2012.

\begin{abstract}
Liu, Z. H., Zhong, S., Stasko, A. K., Edwards, M. C., and Friesen, T. L. 2012. Virulence profile and genetic structure of a North Dakota population of Pyrenophora teres f. teres, the causal agent of net form net blotch of barley. Phytopathology 102:539-546.

A Pyrenophora teres $\mathrm{f}$. teres population in North Dakota was analyzed for virulence variation and genetic diversity using 75 monospore isolates that were collected across a 4-year period (2004 to 2007) from two North Dakota State University agricultural experiment stations at Fargo and Langdon. Pathogenicity tests by inoculation onto 22 barley differential lines at seedling stage revealed 49 pathotypes, indicating a wide range of pathogenic diversity. Two-way analysis of variance of disease ratings revealed a significant difference in the virulence among isolates and in the resistance among barley lines, as well as in the interactions between the two. 'CI5791', 'Algerian', and 'Heartland' were three barley lines

overcome. Forty multilocus genotypes were identified from this set of isolates by genotyping at 13 simple-sequence repeat loci. High percentages of clonal cultures were detected in the samplings from 2005 and 2007 in Fargo and 2005 in Langdon. Using a clone-corrected sample set, the mean gene diversity $(h)$ was estimated to be 0.58 , approximately the same for both locations. The calculated Wright's $F_{S T}$ value is small $(0.11)$ but was significantly $>0$, indicating a significant differentiation between the Fargo and Langdon populations. In the gametic disequilibrium test, only 3 of 78 possible pairwise comparisons over all isolates showed significant $(P<0.05)$ nonrandom association, suggesting a random mating mode. Our results suggest that the populations from the two locations are derived from a common source and undergo frequent recombination. This research provides important information for barley breeders regarding development and deployment of cultivars with resistance to net form net blotch in this region.
\end{abstract} showing a high level of seedling resistance to all North Dakota isolates tested; however, many previously reported resistance genes have been

The ascomycete Pyrenophora teres Drechsler (anamorph: Drechslera teres (Sacc.) Shoemaker) is the causal agent of net blotch of barley, a very common foliar disease in most barleygrowing regions worldwide. The fungus is further classified into two intraspecific taxa, $P$. teres f. teres and $P$. teres f. maculata, which cause net form net blotch (NFNB) and spot form net blotch (SFNB), respectively (40). The two forms have a different global distribution pattern but both can cause significant yield loss and have become increasingly important in recent years $(21,25)$. Breeding and planting of resistant cultivars is often the most economical and environmentally friendly way of combating disease. However, a successful breeding program and utilization of host resistance requires a thorough knowledge of the current virulence and genetic structure of the pathogen population in a particular area.

Variation in virulence among isolates of $P$. teres $\mathrm{f}$. teres has been reported in many regions around the world and many different physiological races or pathotypes have been described using a range of barley differential lines. These reports have included isolates from Australia (11,17,18), New Zealand (7), Canada (44), the United States $(42,50)$, Europe $(5,13-15,36)$, Asia (37), and

Corresponding author: T. L. Friesen; E-mail address: timothy.friesen@ars.usda.gov

* The $\boldsymbol{e}$-Xtra logo stands for "electronic extra" and indicates that the online version contains two supplementary tables.

http://dx.doi.org/10.1094/PHYTO-09-11-0243

This article is in the public domain and not copyrightable. It may be freely reprinted with customary crediting of the source. The American Phytopathological Society, 2012.
Additional keywords: spot form, virulence diversity.
Africa (12). Notably, Steffenson and Webster (42) identified 13 pathotypes from $91 \mathrm{P}$. teres $\mathrm{f}$. teres isolates collected in California. This study also found that isolates collected in Minnesota, Mexico, and England exhibited distinctly different pathotypes, with Mexican pathotypes showing the most similarity to the prevalent California pathotypes. In Western Australia, the pathogen virulence pattern shifted around 1970 when new cultivars were introduced (17) but, during the next 19 years, the virulence among net form isolates remained stable (11). Due to the different sets or numbers of barley differential lines used by individual researchers, comparison of physiological races or pathotypes identified in different studies is difficult. Therefore, suggestions and efforts have been made to identify a set of barley differential lines as a standard for international use $(2,3,42)$; however, this has not yet been universally adopted.

The pathogen's interaction with currently grown cultivars is one of the most important factors influencing its population structure; however, other factors such as sexual recombination, natural mutation, genetic drift, and gene flow also influence the makeup of any given population. $P$. teres has a multifaceted lifestyle, including both pathogenic and saprophytic growth. $P$. teres is able to survive on barley stubble in the field, has a wide host range, and is able to travel long distances through airborne ascospores or infected seed (21). Additionally, P. teres has the capacity to reproduce both sexually and asexually. All of these factors have the potential to significantly influence the genetic structure of a local P. teres population (31).

Molecular markers such as restriction fragment length polymorphism, random amplified polymorphic DNA (RAPD), ampli- 
fied fragment length polymorphism (AFLP), and simple-sequence repeat (SSR) are powerful tools used in analyzing the genetic diversity in fungal pathogen populations, including $P$. teres. The first extensive population genetic analysis for $P$. teres $\mathrm{f}$. teres was done by Peever and Milgroom (31) using RAPD markers to examine five populations originating from Canada, Germany, and the United States. It revealed a Nei's $G_{\text {st }}$ value of 0.46 , indicating that almost $54 \%$ of the total genetic variation exists among different populations, which was higher overall than that for other fungal pathogens. These authors also found that sexual reproduction was present in all but one population. Since this first study, others have continued to investigate the genetic variation and structure of $P$. teres $\mathrm{f}$. teres populations on a local, regional, and global scale $(16,19,20,32,33,38,39)$. In general, molecular markers revealed high allelic and genotypic diversity within populations on all scales. The genetic variation within a population can be distributed in one field (16) or even on one plant (32). Genetic divergence of $P$. teres f. teres was found to be strongly associated with geographic distance(31); therefore, it was speculated that a lower rate or limited gene flow occurred among global or continental populations $(19,31,33)$ whereas, at regional or field levels, genetic exchanges were very likely $(16,20,39)$. The low degree of gametic disequilibrium and the presence of both mating types suggested the relative importance of sexual reproduction in different $P$. teres f. teres populations $(31,33,39)$. However, by analyzing a large number of isolates from different origins, Serenius et al. (38) concluded that genetic similarity between geographic regions and the prevalence of sexual reproduction are highly variable.

North Dakota leads the United States in barley production (28) and NFNB is a common disease in this area $(29,41)$. However, currently only two studies $(31,50)$ have included North Dakota $P$. teres $\mathrm{f}$. teres isolates and these were collected decades ago. No recent systematic study has been undertaken to investigate virulence and genetic structure of the $P$. teres population in North Dakota. In this study, we collected $P$. teres f. teres isolates over four growing seasons from two North Dakota barley-growing regions varying in environmental conditions. Using the collected isolates, we assessed the virulence structure using a set of 22 barley differential lines. Additionally, we evaluated the genetic diversity in the collection and compared the similarity among different populations across two locations and multiple years. The objectives of this study were to identify effective sources of resistance, quantify the genetic variation between and within populations from two locations, and test the hypothesis of random mating within the North Dakota population.

\section{MATERIALS AND METHODS}

Fungal collection and isolation. Leaf samples of 'Hector' with typical NFNB symptoms were collected from North Dakota State University agricultural experiment stations at Fargo and Langdon, ND, in the growing seasons during 2004 to 2007. Fargo and

TABLE 1. Number and designation of Pyrenophora teres f. teres isolates collected in North Dakota

\begin{tabular}{lcl}
\hline Site, year & Number of isolates & \multicolumn{1}{c}{ Designation } \\
\hline $\begin{array}{l}\text { Fargo, ND } \\
2004\end{array}$ & 10 & \\
2005 & 10 & FGOH04Ptt-1 to -10 \\
2006 & 7 & FGOH05Ptt-1 to -10 \\
2007 & $10^{\mathrm{a}}$ & FGOH06Ptt-1 to -7 \\
Langdon, ND & & FGOH07Ptt-1 to -10 \\
2004 & 10 & \\
2005 & 10 & LDNH04Ptt-1 to -10 \\
2006 & 10 & LDNH05Ptt-1 to -10 \\
2007 & $10^{\mathrm{a}}$ & LDNH07Ptt-1 to -10 \\
\hline
\end{tabular}

a One isolate from each of these populations did not produce sufficient levels of inoculum to be included in the virulence tests.
Langdon are located in the southeast and north-central regions, respectively, of North Dakota and are separated by $\approx 300 \mathrm{~km}$. In all, $\approx 25$ symptomatic leaves were randomly collected (two to three spots) within an experimental field plot $\left(\approx 100 \mathrm{~m}^{2}\right.$ in size $)$ at each location to produce our annual isolate collection. The collected leaves were surface sterilized using 6\% sodium hypochlorite for $5 \mathrm{~min}$, rinsed three times with sterilized water, and placed in a petri plate containing $2 \%$ water agar. The plates containing leaf samples were incubated at room temperature on a laboratory bench under constant light. Sporulation from leaf tissue usually began after $\approx 3$ days of incubation. Single conidia were picked under a dissection microscope and transferred to a plate containing $150 \mathrm{ml}$ of V8 juice, $10 \mathrm{~g}$ of Difco potato dextrose agar, $3 \mathrm{~g}$ of $\mathrm{CaCO}_{3}$, and $10 \mathrm{~g}$ of agar dissolved in 1 liter of water to form monoconidial isolates. From each location, 20 to 30 monoconidial isolations were made to construct the annual $P$. teres collection. Up to 10 isolates (Table 1) were randomly selected from the annual collections for each location to conduct virulence tests and SSR marker genotyping.

Virulence test. Selected isolates were evaluated for virulence by inoculating each onto 22 barley lines, including 21 commonly used differential lines for resistance to NFNB and the susceptible check Hector (22) (Fig. 1). Among them, 19 of the differential lines used here were also used by $\mathrm{Wu}$ et al. (50), and lines 'CI9214' and 'Heartland' were used by Tekauz (44). The line 'SM89010' contains at least one dominant gene for resistance to $P$. teres f. teres (10) but, to our knowledge, has not been used previously in a differential set. For each replication, six plants of each line were grown in plastic cones of 25 by $4.5 \mathrm{~cm}$ with three individual plants per cone. All cones were placed into a rack of 98 with the barley 'Robust' planted as a border. The plants were grown in the greenhouse and inoculated at the two- to three-leaf stage with the second leaf fully expanded. Fungal inoculum preparation, inoculation, and post-inoculation incubation followed the same procedure described by Abu Qamar et al. (1). Inoculations were conducted at least three times to show consistent characterization of disease reaction for each isolate. One isolate in 2006 from the Fargo location was identified as spot form (P. teres f. maculata) (23), and two isolates in 2007 (one from Fargo and the other from Langdon) did not produce enough inoculum to be included in the virulence tests (Table 1). The isolates $0-1$ and $15 \mathrm{~A}$, originally collected in Ontario, Canada (47) and Solano County, CA (42), respectively, were included as reference isolates. Disease was evaluated 7 days after inoculation using the 1to-10 rating scale described by Tekauz (43), with 1 being highly resistant and 10 being highly susceptible. The average from three replications showing similar reaction was used to determine the virulence of a particular isolate on a particular line. Average disease reactions $<5.0$ were considered to be low virulence and $\geq 5.0$ were considered to be high virulence on each differential line (44). The numerical data were transformed into 0 as low virulence and 1 as high virulence for calculating the number and diversity of pathotypes.

Molecular marker and mating type gene analysis. In all, $\approx 40$ pairs of SSR primers harboring trinucleotide or dinucleotide microsatellites have been developed from the recently completed $P$. teres f. teres genome sequence and used in genetic linkage mapping of the fungal genome (9). Among these SSRs, 13 primer pairs produced quality polymorphic markers from loci that mapped across the genome and, therefore, were chosen for genotyping the fungal isolates used in this study. The sequences for each primer pair are listed in Table 2. In our experiment, an M13 tail sequence (CACGACGTTGTAAAACGAC) was added on the 5 ' end of all forward primers so that we could perform the electrophoresis and visualization on a LICOR-4300 genotyping system (Li-Cor Bioscience). For each isolate, DNA was extracted from the ground powder of young mycelial tissue using a BioSprint 15 instrument (Qiagen) with the corresponding kit 
(Qiagen). Polymerase chain reaction (PCR) setup, amplification conditions, and electrophoresis were the same as those described by Zhong et al. (53). SSR alleles were scored manually as sized fragments with reference to a 50-bp ladder (Li-Cor Bioscience) and, for each SSR primer pair, amplicons of the same size were considered to be the same allele. In SSR genotyping, two P. teres f. teres reference isolates, $15 \mathrm{~A}$ and $0-1$, and two $P$. tritici-repentis isolates, 86-124 and Pti2, were also included. The raw data were then transformed into letter designations (A, B, C, and so on) as different alleles for population genetic analysis.

To investigate the distribution of the two mating types, MATspecific SNP primers developed by Lu et al. (24) for P. teres f. teres were used to amplify mating type genes from all isolates. The PCR was set up and performed according to the conditions described by $\mathrm{Lu}$ et al. (24). The PCR products were separated on a 1\% agarose gel and scored as PttMAT1-1 (1,143 bp) and PttMAT1-2 (1,421 bp) based on the sizes reported by Lu et al. (24).

Statistical and population genetic analyses. Two-way analysis of variance (ANOVA) for disease reaction of three replications was performed using Microsoft Excel 2003 to test the hypothesis that a significant difference exists among the isolates, barley lines, and isolate-line interactions. The isolates were combined as different populations based on the times and locations they were collected. The Nei's unbiased gene diversity $(h)(30)$ for different populations was estimated using POPGENE version 1.32 (51). The number of pathotypes and genotypes and the genotype diversity for each population were calculated using Multilocus 1.3 (4). The diversity is defined as the probability that two individuals taken at random have different genotypes or pathotypes. This is equivalent to $[n /(n-1)]\left(1-\Sigma p_{i}^{2}\right)$, where $p_{i}$ is the frequency of the $i$ th genotype or pathotype and $n$ is the number of individuals sampled. This value is 0 if every individual is the same and 1 if every individual is different. The population genetic differentiation was estimated based on Wright's $F_{S T}(\theta)$ using Multilocus 1.3 (4). The null hypothesis of no differentiation between two populations was tested by comparing the observed $\theta$ to that obtained from 1,000 artificially randomized datasets. Similar measures of population differentiation with a correction for sampling size, including $G_{S T}^{\prime}$ and $G_{S T}^{\prime \prime}(26)$, were also calculated using
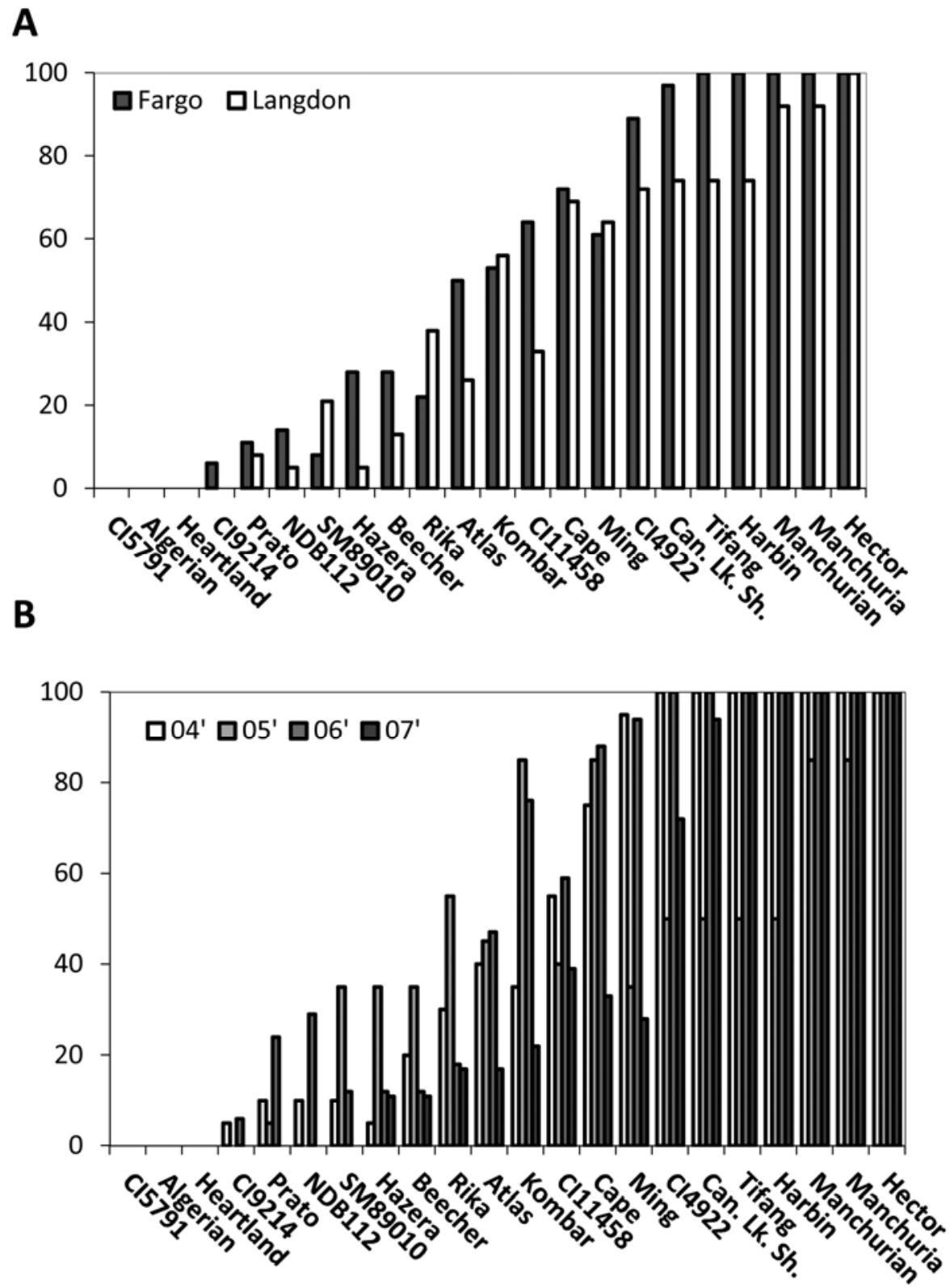

Fig. 1. Percentage of North Dakota Pyrenophora teres f. teres isolates virulent on the barley differential lines used. Isolates were pooled by A, location (Fargo and Langdon) and $\mathbf{B}$, year. 
the computer software GenoDive (27). The isolates with redundant genotypes were removed from the collection to evaluate the gametic disequilibrium. The web-based program Genepop on the Web (http://genepop.curtin.edu.au/) (35) was used to test whether nonrandom association occurs between SSR loci based on the calculated $P$ value.

\section{RESULTS}

Pathotype and virulence spectrum of $P$. teres f. teres. The disease means of 75 isolates on 22 lines from three replications are shown in Supplementary Table 1. The grand mean of the different isolates ranged from 2.6 (LdnH05Ptt-4) to 6.2 (FgoH06Ptt-4) and the grand mean of the different lines ranged from 1.2 (CI5791) to 8.4 (Hector). The ANOVA showed that differences in the virulence of isolates, resistance of differential lines, and isolate-line interactions were significant $(P<0.05)$ (Table 3).

Based on the reaction to 22 barley lines, 49 pathotypes were detected from 75 isolates resulting in an average of 0.65 pathotypes/isolate (Table 4). High virulence diversity (0.98) was revealed in the total isolates collected from the 4 years and two locations, with the two locations having the same level of virulence diversity (Table 4). Although a slightly lower level of diversity was observed in some years, isolates could not be grouped by year and location based on pathotype (Supplementary Table 2). For example, the frequency of the most common pathotype was 6 and it included isolates from both locations spanning 3 years (FgoH04Ptt-7, FgoH04Ptt-9, LdnH04Ptt-3,
LdnH06Ptt-4, LdnH06Ptt-5, and LdnH07Ptt-8). The least virulent isolate was LdnH05Ptt-4, which showed low virulence on all the barley lines except susceptible check Hector. The most virulent isolate was FgoH06Ptt-4, which had high virulence on 17 of 22 lines.

The percentage of virulent isolates on individual lines is shown in Figure 1 based on location and year. In general, the Fargo location had a higher percentage of virulent isolates on all lines except SM89010 and 'Rika' (Fig. 1A). The isolates from the 2005 collection had a higher percentage of isolates with virulence on SM89010, Rika, 'Hazera', and 'Beecher' but a lower percentage of isolates with virulence on 'Ming', 'Tifang', 'Harbin', 'Canadian Lake Shore' (CLS), and 'CI4922', which are of Chinese origin or derived from a Chinese cultivar (Fig. 1B). For the two reference isolates $15 \mathrm{~A}$ and $0-1,15 \mathrm{~A}$ was only virulent on lines Beecher, 'Atlas', Hazera, 'Prato', and Hector, whereas 0-1 showed virulence on all those lines except for Prato but additionally was virulent on Ming, Tifang, Harbin, CLS, and CI4922.

Among the 22 barley lines, only CI5791, 'Algerian', and Heartland were resistant to all 75 isolates tested. In contrast, CI4922, CLS, Tifang, Harbin, 'Manchurian', and 'Manchuria' were susceptible to all isolates from at least 1 year of collections for both locations, indicating that the resistance in those lines has been overcome by the North Dakota $P$. teres f. teres population (Fig. 1B). Lines CI9214, Prato, SM89010, 'NDB112', Rika, Beecher, and Atlas were susceptible to $<50 \%$ of the isolates regardless of the year and location, particularly the first four (CI9214, Prato, SM89010, and NDB112), which were susceptible to $<10 \%$ of the total isolates tested (Fig. 1A and B). The reaction

TABLE 2. Primer information and total number of simple-sequence repeat (SSR) alleles used in the genotyping of the Pyrenophora teres f. teres populations

\begin{tabular}{|c|c|c|c|c|c|}
\hline ID & $\begin{array}{c}\text { Map } \\
\text { location }^{\mathrm{a}}\end{array}$ & Forward primer & Reverse primer & $\begin{array}{l}\text { Size } \\
(b p)^{b}\end{array}$ & $\begin{array}{c}\text { Number of } \\
\text { alleles }\end{array}$ \\
\hline (TGT)12_243004 & LG2.1 & GTTGGAAGGCCATAAGATGC & AATTGGAAGTCATAGCGACACA & 201 & 5 \\
\hline (CGA)24_153210 & LG10 & CAGAGGATAGCGTGTTTGAGG & GCCATTGCCCTCGACTAA & 205 & 4 \\
\hline (CTG)19_61882 & LG7 & CCGGATTCATACCCACTATGTT & CGCCTTCATATAACGAGACTACA & 196 & 9 \\
\hline (CTG)15_105102 & LG3 & CACATCACAAAGGCGGTTT & GACGGTGCATTGATTCGTT & 215 & 7 \\
\hline (TGT)32_243145 & LG3 & ACTGTTGAGGTGCTGAAGATGA & CAGATTGGACGACAAGACTCAC & 262 & 9 \\
\hline (GTG)17_243085 & LG22 & GATGCCAGAAGAATGGTAGAAGA & AGTAGCACAAGCGAACAAGATG & 166 & 5 \\
\hline (CAT)13_76865 & LG26 & TTAGATCGCTCAAGAAGGAATG & ATTATCAAGTATCGCCGTCATC & 228 & 7 \\
\hline (GAT)13_245900 & LG15 & CCGGCCACCTACTTACTTAGATT & GCGACTTACATCTGCATACCC & 251 & 4 \\
\hline (GTC)38_165205 & LG9 & ATCCGAACCGCTCTCCTT & GCAGGTGCTCGTACAAATATC & 268 & 7 \\
\hline (ACA)14_245269 & LG24 & CGCTGGTACATCTTCATTTCAG & CGCATAGTTGGAGTGTGGATAG & 194 & 4 \\
\hline (AC)12_192737 & LG4 & TGTAGATGCGAGTGCGGGTA & GGAATCTTTTCATCCAATTGC & 244 & 10 \\
\hline (AAC) $10 \_189811$ & LG3 & TACTTAAGCATTCGAAGCCG & AGCAGCGTTCTTGAGGAAGA & 225 & 6 \\
\hline (AC)13_137091 & LG20 & TATCGGCGTTCTTGATGTGTC & ATTGCTTGTTGCTAGTCACCG & 231 & 7 \\
\hline
\end{tabular}

${ }^{a}$ Refer to Ellwood et al. (9) for linkage group (LG) location of each SSR marker; three on LG3 were separated by 50 centimorgans.

b Size was determined using the genome sequence of $P$.teres f.teres isolate $0-1$.

TABLE 3. Analysis of variance for disease reactions induced by 75 Pyrenophora teres f. teres isolates from North Dakota on a set of 22 barley differentials

\begin{tabular}{|c|c|c|c|c|c|}
\hline Source of variation & df & MS & $F$ & $P$ value & $F$ crit \\
\hline Isolates & 74 & 52.83 & 30.41 & 0 & 1.29 \\
\hline Lines & 21 & 918.56 & 528.65 & 0 & 1.56 \\
\hline Interaction & 1,554 & 5.63 & 3.24 & $3.1 \mathrm{E}-176$ & 1.07 \\
\hline Within & 3,300 & 1.74 & $\ldots$ & $\ldots$ & $\ldots$ \\
\hline
\end{tabular}

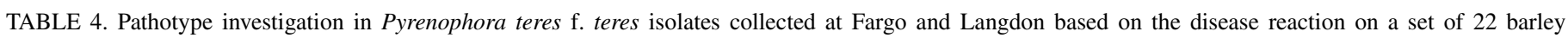
differentials ${ }^{\mathrm{a}}$

\begin{tabular}{|c|c|c|c|c|c|c|c|c|c|c|c|c|}
\hline \multirow[b]{2}{*}{ Year } & \multicolumn{4}{|c|}{ Fargo } & \multicolumn{4}{|c|}{ Langdon } & \multicolumn{4}{|c|}{ Total } \\
\hline & Isolates & Pathotypes & Frequency & Diversities & Isolates & Pathotypes & Frequency & Diversities & Isolates & Pathotypes & Frequency & Diversities \\
\hline 2004 & 10 & 9 & 2 & 0.97 & 10 & 9 & 2 & 0.98 & 20 & 17 & 3 & 0.98 \\
\hline 2005 & 10 & 7 & 4 & 0.86 & 10 & 7 & 4 & 0.86 & 20 & 14 & 4 & 0.94 \\
\hline 2006 & 7 & 7 & 1 & 1.00 & 10 & 9 & 2 & 0.98 & 17 & 15 & 2 & 0.99 \\
\hline 2007 & 9 & 4 & 3 & 0.80 & 9 & 9 & 1 & 1.00 & 18 & 11 & 4 & 0.92 \\
\hline Total & 36 & 27 & 4 & 0.98 & 39 & 29 & 4 & 0.98 & 75 & 49 & 6 & 0.98 \\
\hline
\end{tabular}

a Isolates = number of isolates, Pathotypes = number of pathotypes, and Frequency = maximum frequency. 
spectrum between CI4922, CLS, Tifang, and Harbin was very similar, as was that between Manchurian and Manchuria.

Genetic diversity, structure, and differentiation of $\boldsymbol{P}$. teres $\mathbf{f}$. teres. The SSR primer pairs used produced variable numbers of alleles ( $n=4$ to 10 ), and 84 alleles were generated from the 13 loci, with an average of 6.5 alleles/locus (Table 2). The size, primer sequence, and linkage group location for each SSR locus are listed in Table 2. Based on the 13 loci, 40 genotypes were detected from 77 isolates having an overall genotype diversity of 0.96 and a mean gene diversity of 0.62 (Table 5). Fargo 2005, Fargo 2007, and Langdon 2005 isolates had only one or two genotypes, indicating the clonality of the cultures; however, no genotypes were shared among the two locations or the different years. Fargo and Langdon had 15 and 25 unique genotypes, respectively. Because fewer clonal isolates were identified, the Langdon collection had a relatively higher mean gene diversity than Fargo (0.61 versus 0.53 ). However, their mean gene diversity values were approximately equal $(0.58)$ if the clone-corrected sample set was used (Table 5).

To determine the genetic differentiation among $P$. teres $\mathrm{f}$. teres populations collected in different year or location, the Wright's $F_{S T}$ value (48) was estimated using software Multilocus 1.3. The $\theta$ values were 0.11 to 0.50 (Table 6 ) and all of these values were shown to be significantly different than zero $(P<0.001)$, rejecting the null hypothesis that no differentiation exists among the populations. The highest $F_{S T}(0.50)$ was observed among populations collected in different years from Fargo and, when comparing isolates from Fargo and Langdon (Fgo_all versus Ldn_all), an $F_{S T}$ of 0.11 was obtained. The population differentiation between two locations was also estimated using two unbiased forms of measurement, including $G_{S T(N e i)}^{\prime}$ and $G^{\prime \prime}{ }_{S T}$. The $G_{S T \text { (Nei) }}^{\prime}$ value was calculated as 0.118 , which is similar to the $F_{S T}$, while a higher value ( 0.283$)$ was obtained for $G^{\prime \prime}{ }_{S T}$.

The distribution of the mating type genes was highly variable in different years and locations, with MAT1-1 occurring in a higher frequency overall (PttMAT1-1:PttMAT1-2 = 52:24) (Table 5). However, with a clone-corrected data set, the ratio of PttMAT11:PttMAT1-2 was $23: 17$, which fits a $1: 1$ ratio $(P=0.34)$, indi-

TABLE 5. Summary of population genetic analysis information comparing Pyrenophora teres f. teres populations from the Fargo and Langdon locations

\begin{tabular}{lccl}
\hline & \multicolumn{3}{c}{ Population } \\
\cline { 2 - 4 } Statistics & Fargo & Langdon & Total \\
\hline Number of isolates & 37 & 40 & 77 \\
Number of genotypes & 15 & 25 & 40 \\
Mating type gene ratio & & & \\
$\quad$ All samples & $25: 11$ & $27: 13$ & $52: 24$ \\
$\quad$ Clone-corrected samples & $6: 9$ & $17: 8$ & $23: 17^{\mathrm{b}}$ \\
$\quad$ Mean allelic diversity $(h)^{\mathrm{c}}$ & & & \\
$\quad$ All samples & 0.53 & 0.61 & 0.62 \\
$\quad$ Clone-corrected samples & 0.58 & 0.58 & 0.61 \\
Genotype diversity & & & \\
$\quad$ All samples & 0.87 & 0.95 & 0.96 \\
Clone-corrected samples & 1.00 & 1.00 & 1.00 \\
\hline
\end{tabular}

a PttMAT1-1:PttMAT1-2.

b The ratio was estimated to fit $1: 1(P=0.34)$.

${ }^{c}$ The mean gene diversity $(h)$ was estimated based on Nei (30).

TABLE 6. Genetic differentiation $\left(F_{S T}\right)$ among Pyrenophora teres f. teres populations collected in different years or locations

\begin{tabular}{lcc}
\hline Population differentiation & $F_{S T}(\theta)$ & $P$ value \\
\hline FGO_all vs. LDN_all & 0.11 & $<0.001$ \\
Among 4 years in Fargo & 0.52 & $<0.001$ \\
Among 4 years in Langdon & 0.30 & $<0.001$ \\
Among 4 years all & 0.20 & $<0.001$ \\
Among 8 all & 0.12 & $<0.001$ \\
\hline
\end{tabular}

cating approximately equal distribution of both mating types. The test for gametic disequilibrium among the 13 SSR loci was conducted using isolates without redundant genotypes from Fargo and Langdon separately or combined. Of 78 possible pairwise comparisons, only two pairs in the Fargo population and seven pairs in the Langdon population showed nonrandom association at the $95 \%$ confidence level, and no common pair was found in both populations (Table 7). The SSR pairs showing gametic disequilibrium involved all SSR loci except (GAT)13_245900 and (AAC)10_189811. In the combined analysis, only three of them remained significant (Table 7). The three SSR loci (CTG)15_105102, (TGT)32_243145, and (AAC)10_189811, located in the same linkage group (LG3), did not show nonrandom association, indicating that they were separated by long genetic distances, which correlates with our genetic linkage mapping results (9).

\section{DISCUSSION}

Our study revealed a high level of diversity for both virulence and molecular markers. In all, 49 pathotypes (0.65 pathotype/ isolate) were detected in the virulence test by inoculation onto the 22 barley differential lines. This level of virulence diversity was higher than that reported by Steffenson and Webster (42) in California or by Tekauz (44) in western Canada, where 13 of 91 pathotypes (0.14 pathotype/isolate) and 45 of 182 pathotypes (0.24 pathotype/isolate) were identified, respectively. A high level of virulence diversity was also reported in other studies. For example, 15 pathotypes were identified by Wu et al. (50) from a collection of 23 isolates that had diverse geographic origins. Jonsson et al. (15) reported 12 pathotypes from 25 Swedish isolates. Douiyssi et al. (8) reported that, of 15 isolates collected from Morocco, none was identical in virulence on 38 barley lines. Cromey and Parkes (7) identified 11 pathotypes among 29 New Zealand isolates using 31 barley lines. The number of pathotypes or the level of virulence diversity was largely dependent on the number and differentiating ability of the set of barley lines used. However, researchers have used different numbers and sets of differential lines, making it difficult to compare the pathotype diversity among different studies.

We used 22 barley differential lines which included 17 and 19 of the lines used by Steffenson and Webster (42) and Wu et al. (50), respectively and, thus, we could compare the pathotypes identified in this study with those reported by these previous studies. In contrast to the California population, with $80 \%$ of the isolates virulent on Prato and only $1 \%$ of the isolates virulent on lines having Chinese origin or background, including Manchurian, Manchuria, CI4922, and Harbin (42), the majority of the North Dakota isolates showed low virulence on Prato but high virulence on lines with Chinese origin or Chinese-derived lines. One Minnesota isolate (MN1A) reported by Steffenson and

TABLE 7. Summary of the pairs of simple-sequence repeat (SSR) loci that show evidence of linkage disequilibrium at $P<0.05$ in the Fargo and Langdon populations

\begin{tabular}{lll}
\hline Population & \multicolumn{1}{c}{ SSR pairs } & $P$ value \\
\hline Fargo & (CGA)24_153210 and (ACA)14_245269 & 0.0349 \\
& (CAT)13_76865 and (AC)13_137091 & 0.0499 \\
Langdon & (GTC)38_165205 and (CTG)15_105102 & 0.0157 \\
& (CTG)19_61882 and (CGA)24_153210 & 0.0120 \\
& (CTG)19_61882 and (TGT)12_243004 & 0.0340 \\
& (CAT)13_76865 and (ACA)14_245269 & 0.0205 \\
& (TGT)32_243145 and (ACA)14_245269 & 0.0045 \\
& (GTG)17_243085 and (AC)12_192737 & 0.0314 \\
Total & (CTG)15_105102 and (AC)12_192737 & 0.0144 \\
& (CAT)13_76865 and (ACA)14_245269 & 0.0468 \\
& (TGT)32_243145 and (ACA)14_245269 & 0.0075 \\
& (CTG)15_105102 and (AC)12_192737 & 0.0183 \\
\hline
\end{tabular}


Webster (42) is similar to most of the North Dakota isolates in virulence on lines of Chinese origin as well as Prato. This is most likely due to their relatedness in physical distance. Based on the similarity of the reaction to the 22 lines, Wu et al. (50) grouped one North Dakota isolate (ND89-19) with isolates from Minnesota, Montana, and Canada but not with the majority of the California isolates or those collected from other countries. Two of our isolates, LdnH06Ptt-9 and LdnH07Ptt-7, have a virulence pattern identical to that of ND89-19 on the 19 lines that were in common. Tekauz (44) used only nine barley lines as differentials, with three of the lines being in common with our lines (CI5791, Heartland, and CI9214). By comparison of virulence on CI5791, Heartland, and CI9214, western Canada had a higher percentage of virulent isolates than North Dakota (4, 52, and 74 versus 0,0 , and 3\%). Interestingly, the reference isolate $0-1$, collected from Ontario, Canada, had the same virulence pattern as North Dakota isolates on Prato and the Chinese-derived lines. Based on the reactions of common barley lines, we conclude that the majority of the North Dakota $P$. teres f. teres isolates are also different in their virulence pattern than those collected in other geographical regions $(2,7$, $8,11,13,15)$.

Three barley lines (CI5791, Heartland, and Algerian) were highly resistant at the seedling stage to all of the North Dakota isolates as well as to the P.teres f. teres reference isolates tested, indicating that they are potential sources of NFNB seedling resistance in North Dakota. Other lines, including CI9214, Hazera, SM89010, Prato, NDB112, Beecher, and Rika, were susceptible to $<20 \%$ of the North Dakota isolates and, therefore, could be used in combination with other resistance sources to enhance the durability of resistant cultivars. The resistance found in 'CI5791', an Ethiopian-derived two-row barley, was also effective against almost all the isolates from Finland and Lithuania $(13,36)$. However, virulence on CI5791 has been reported in Russia (2,3), Canada (44), and other countries (3). Unfortunately, CI5791 is less resistant to SFNB (45) (Friesen et al., unpublished data). Heartland (PI552963) is a six-rowed spring barley cultivar developed in Canada that also harbors resistance to Puccinia graminis (46) but it has not been widely used in Pyrenophora teres $\mathrm{f}$. teres virulence test studies. It is possible that this line could prove valuable in breeding cultivars with resistance to both diseases. NDB112 (CIho 11531) is a North Dakota breeding line selected as a resistance source effective against spot blotch caused by Cochliobolus sativus (49). It was resistant to ND89-19 reported by Wu et al. (50) and to $80 \%$ of the isolates we collected. Some isolates from Fargo 2004, Fargo 2006, and Langdon 2006 were virulent on this line but with average infection types just above 5.0; therefore, it could still serve as an important source of partial resistance in the field. Other resistant lines reported elsewhere, such as 'CI9819' (3,36,37), 'c-8755', and 'CI 9825' (3), should be tested with our collection to expand the resistance base of North Dakota barley. In some cases, seedling resistance has been effective in the adult plant stage; however, this is not always the case (21). Therefore, barley lines showing seedling resistance also need to be tested at the adult plant stage for resistance to the local pathogen population.

High levels of genetic diversity of $P$. teres f. teres were also revealed in this North Dakota population using molecular markers. We obtained a value of 0.62 for gene diversity over all isolates and all loci. Peever and Milgroom (31) analyzed five populations using 10 RAPD loci and found that gene diversity in each population ranged from 0.08 in North Dakota to 0.17 in New York, with the mean gene diversity over all populations at 0.12 . Jonsson et al. (16) reported a mean gene diversity (averaged over 19 RAPD markers) of 0.236 and 0.253 using isolates collected from two fields in western Sweden located $20 \mathrm{~km}$ apart. By comparison, our study revealed relatively higher gene diversity, which may be due to the SSR marker system we used (that is, a multiallelic marker). In addition, SSR loci usually produce a higher level of polymorphism than most other markers. In this study, individual SSRs revealed as many as 10 alleles with an average of 6.5 alleles/locus. This set of primers provides a powerful tool for population studies of $P$. teres, including $P$. teres $\mathrm{f}$. maculata.

Using 13 SSR loci, we also detected a high genotype diversity (Table 5) in all populations, with the exception of Fargo 2005, Fargo 2007, and Langdon 2005, where a clonal or near clonal population was present. The lack of diversity in Fargo 2005, Fargo 2007, and Langdon 2005 is not likely due to the sampling because this was done by a very similar process each year; therefore, it is most likely due to asexual reproduction in a large area within a field. In total, 40 genotypes were obtained, which in some ways agrees with the 49 pathotypes we identified. In most cases, the isolates having the same genotype tend to have a similar virulence pattern on the 22 differential lines. For example, all isolates collected in Fargo 2007 had the same genotype and consisted of four pathotypes. These four pathotypes only differ in their virulence to three lines in which intermediate disease reactions were recorded. Therefore, the difference of these pathotypes is largely due to the artificial cutoff points of 5.0. We adopted the same cutoff point as described by Tekauz (44) to separate isolates with low and high virulence because the infection type of 5.0 exhibited considerable chlorosis, which surrounded necrotic netlike lesions and is indicative of host susceptibility. However, some isolates having the same or a similar genotype showed significant differences in the disease reaction on certain lines. One possibility for phenotypic differences in genotypically similar or identical isolates is that single genetic mutations could lead to changes in virulence among isolates without affecting the SSR markers used for genotyping. Alternatively, this might be due to the fact that genes controlling virulence are not tightly linked with the SSR markers used; furthermore, the frequent sexual reproduction of the fungus in this area (see below) can break the association of SSR loci with virulence genes.

A small $F_{S T}$ value (0.11) was obtained when we compared the isolates collected from Fargo and Langdon, indicating that a large portion of the genetic differentiation occurred within the two locations rather than between the two locations. The similarity of $P$. teres f. teres isolates from the two locations was also made evident by the fact that the virulence pattern from the two locations was very similar (Fig. 1A). However, the statistical analysis indicated that isolates from the two locations were significantly differentiated. Furthermore, the differentiation value increased when using methods with a correction of sampling size, suggesting that the two populations could be more distant genetically. The comparison also showed a relatively higher $F_{S T}$ value among populations collected in different years than from populations identified by location. This would indicate either that the North Dakota $P$. teres f. teres population changes over time or that the estimate of $F_{S T}$ among years is biased due to the small sample size. Leišova et al. (20) analyzed 37 isolates from different locations of the Czech Republic and found that the variability seemed to have been influenced more by the year of sampling than by the origin of the isolates. Other studies also found no or very low genetic differentiation among locations within a region or country but high differentiation among populations originating from different countries or continents $(19,31,38)$.

In gametic disequilibrium analysis, we detected only a very low percentage of the SSR pairs showing significant nonrandom association in both Fargo and Langdon populations (2.5 and 8.9\%, respectively), suggesting frequent occurrence of sexual reproduction of $P$. teres $\mathrm{f}$. teres in North Dakota. This result agrees with the previous study done by Peever and Milgroom (31), who genetically analyzed 35 isolates collected in 1991 in North Dakota and concluded that the North Dakota P. teres f. teres population appeared to be mating randomly. The random mating mode is also supported in this study by the presence of both 
mating types with approximately equal distribution and the significant differentiation between population collected from different years and locations. Sexual reproduction of $P$. teres $\mathrm{f}$. teres was also evident from genetic population analysis in many other areas worldwide $(16,31,34,39)$. Our data indicate the occurrence of sexual reproduction based on gametic disequilibrium analysis using SSR markers but we also observed a high percentage of monoclonal isolates in Fargo 2005, Fargo 2007, and Langdon 2005, indicating that asexual reproduction during the growing season is common. It should also be pointed out that the interpretation of gametic disequilibrium must be taken with caution when the sample size is $<100(6,52)$. Lehmensiek et al. (19) investigated the population structure of $P$. teres $f$. teres in Australia and South Africa using AFLP markers and concluded that the fungus mainly reproduces asexually. Based on the analysis of diverse populations from different geographic regions, Serenius et al. (38) concluded that prevalence of the sexual stage is highly variable and dependent on environmental conditions and cultural practices. Therefore, a systematic and large area of sampling is needed to provide solid evidence on the prevalence of the sexual stage.

This is the first systematic study of a P. teres f. teres population in North Dakota; however, due to the small sample sizes of the separate populations, the population genetic analysis is somewhat limited. Therefore, our interpretation based on those indexes is preliminary and should be taken as such. Despite the small sample size and a small sampling area, we still detected a large number of pathotypes and genotypes, indicating the diversity of the North Dakota $P$. teres f. teres population. Therefore, barley breeding for NFNB resistance in North Dakota should employ a strategy of pyramiding as many effective resistance genes as possible. Alternatively, it is likely that susceptibility genes with corresponding necrotrophic effectors (host-selective toxins) produced by the pathogen are involved, as they are in Rika and 'Kombar' (1). If so, these genes will need to be removed in order to increase the level of resistance to this destructive pathogen. We also found that the fungal populations in Fargo and Langdon are similar in virulence toward barley lines at seedling stages; therefore, the cultivars with the same seedling resistance gene can be deployed in the Fargo and Langdon regions. Finally, the fungal populations at both locations should be constantly monitored due to prominent sexual reproduction that may bring new virulent pathotypes.

\section{ACKNOWLEDGMENTS}

This research was supported by USDA-ARS CRIS Project 544222000-043-00D, USDA-NIFA-AFRI Triticeae Coordinated Agricultural Project (T-CAP) Competitive Grant 2011-68002-30029 and The American Malting Barley Association. We thank D. Holmes for technical support.

\section{LITERATURE CITED}

1. Abu Qamar, M., Liu, Z. H., Faris, J. D., Chao, S., Edwards, M. C., Lai, Z., Franckowiak, J. D., and Friesen, T. L. 2008. A region of barley chromosome $6 \mathrm{H}$ harbors multiple major genes associated with net type net blotch resistance. Theor. Appl. Genet. 117:1261-1270.

2. Afanasenko, O. S., Hartleb, H., Guseva, N. N., Minarikova, V., and Janosheva, M. 1995. A set of differentials to characterize populations of Pyrenophora teres Drechs. for international use. J. Phytopathol. 143:501507.

3. Afanasenko, O. S., Jalli, M., Pinnschmidt, H. O., Filatova, O., and Platz, G. J. 2009. Development of an international standard set of barley differential genotypes for Pyrenophora teres f. teres. Plant Pathol. 58:665676.

4. Agapow, P.-M., and Burt, A. 2000. Indices of multilocus linkage disequilibrium. Mol. Ecol. Notes 1:101-102.

5. Arabi, M. I. E., Al-Safadi, B., and Charbaji, T. 2003. Pathogenic variation among isolates of Pyrenophora teres, the causal agent of barley net blotch. J. Phytopathol. 151:376-382.
6. Brown, A. H. D. 1975. Sample size required to detect linkage disequilibrium between two or three loci. Theor. Popul. Biol. 8:184-201.

7. Cromey, M. G., and Parks, R. A. 2003. Pathogenic variation in Dreschlera teres in New Zealand. N. Z. Plant Prot. 56:251-256.

8. Douiyssi, A., Rasmusson, D. C., and Roelfs, A. P. 1998. Responses of barley cultivars and lines to isolates of Pyrenophora teres. Plant Dis. 82:316-321.

9. Ellwood, S. R., Liu, Z. H., Syme, R. A., Lai, Z., Hane, J. K., Keiper, F., Moffat, C. S., Oliver, R. P., and Friesen, T. L. 2010. A first genome assembly of the barley fungal pathogen Pyrenophora teres f. teres. Genome Biol. 11:R109.

10. Friesen, T. L., Faris, J. D., Lai, Z., and Steffenson, B. J. 2006. Identification and chromosomal location of major genes for resistance to Pyrenophora teres in a doubled-haploid barley population. Genome 49:855-859.

11. Gupta, S., and Loughman, R. 2001. Current virulence of Pyrenophora teres on barley in Western Australia. Plant Dis. 85:960-966.

12. Harrabi, M., and Kamel, A. 1990. Virulence spectrum to barley in some isolates of Pyrenophora teres from Mediterranean region. Plant Dis. $74: 230-232$.

13. Jalli, M. 2004. Suitability of a selected barley differential set for Pyrenophora teres f. teres virulence screening. Pages 266-269 in: Proc. 9th Int. Barley Genet. Symp. Brno, Czech Republic.

14. Jalli, M., and Robinson, J. 2000. Stable resistance in barley to Pyrenophora teres f. teres isolates from the Nordic-Baltic region after increase on standard host genotypes. Euphytica 113:71-77.

15. Jonsson, R., Bryngelsson, T., and Gustafsson, M. 1997. Virulence studies of Swedish net blotch isolates Drechslera teres and identification of resistant barley lines. Euphytica 94:209-218.

16. Jonsson, R., Sall, T., and Bryngelsson, T. 2000. Genetic diversity for random amplified polymorphic DNA (RAPD) markers in two Swedish populations of Pyrenophora teres. Can. J. Plant Pathol. 22:258-264.

17. Khan, T. N. 1982. Changes in barley genotypes grown in Western Australia. Plant Dis. 66:655-656.

18. Khan, T. N., and Boyd, W. J. R. 1969. Physiologic specialization in Drechslera teres. Aust. J. Biol. Sci. 22:1229-1235.

19. Lehmensiek, A., Bester-van der Merwe, A. E., Sutherland, M. W., Platz, G., Kriel, W. M., Potgieter, G. F., and Prins, R. 2010. Population structure of South African and Australian Pyrenophora teres isolates. Plant Pathol. 59:504-515.

20. Leišova, L., Minarı̌́́ova, V., Kučera, L., and Ovesná, J. 2005. Genetic diversity of Pyrenophora teres isolates as detected by AFLP analysis. J. Phytopathol. 153:569-578.

21. Liu, Z. H., Ellwood, S. R., Oliver, R. P., and Friesen, T. L. 2011. Pyrenophora teres: Profile of an increasingly damaging barley pathogen. Mol. Plant Pathol. 12:1-19.

22. Liu, Z. H., Faris, J. D., Edwards, M. C., and Friesen, T. L. 2010. Development of expressed sequence tag (EST)-based markers for genomic analysis of a barley $6 \mathrm{H}$ region harboring multiple net form net blotch resistance genes. Plant Genome 3:41-52.

23. Liu, Z. H., and Friesen, T. L. 2010. Identification of Pyrenophora teres $\mathrm{f}$. maculata, causal agent of spot type net blotch in North Dakota. Plant Dis. 94:480.

24. Lu, S., Platz, G. J., Edwards, M. C., and Friesen, T. L. 2010. Mating type locus-specific polymerase chain reaction markers for differentiation of Pyrenophora teres f. teres and P. teres f. maculata, the causal agents of barley net blotch. Phytopathology 100:1298-1306.

25. McLean, M. S., Howlett, B. J., and Hollaway, G. J. 2009. Epidemiology and control of spot form of net blotch Pyrenophora teres f. maculata of barley: A review. Crop Pasture Sci. 60:303-315.

26. Meirmans, P. G., and Hedrick, P. W. 2011. Assessing population structure: $F_{S T}$ and related measures. Mol. Ecol. Resour. 11:5-18.

27. Meirmans, P. G., and VanTienderen, P. H. 2004. Genotype and Genodive: Two programs for the analysis of genetic diversity of asexual organism. Mol. Ecol. Notes 4:792-794.

28. NASS. 2010. Small grain 2010 summary September 2010. USDA National Agricultural Statistics Service.

29. Neate, S., and McMullen, M. 2005. Barley Disease Handbook. North Dakota State Barley Council and North Dakota State University.

30. Nei, M. 1973. Analysis of gene diversity in subdivided populations. Proc. Natl. Acad. Sci. USA 70:3321-3323.

31. Peever, T. L., and Milgroom, M. G. 1994. Genetic structure of Pyrenophora teres populations determined with random amplified polymorphic DNA markers. Can. J. Bot. 72:915-923.

32. Peltonen, S., Jalli, M., Kammiovirta, K., and Karjalainen, R. 1996. Genetic variation in Drechslera teres populations as indicated by RAPD markers. Ann. Appl. Biol. 128:465-477.

33. Rau, D., Brown, A. H. D., Brubaker, C. L., Attene, G., Balmas, V., Saba, E., and Papa, R. 2003. Population genetic structure of Pyrenophora teres Drechs. the causal agent of net blotch in Sardinian landraces of barley 
Hordeum vulgare L. Theor. Appl. Genet. 106:947-959.

34. Rau, D., Maier, F. J., Papa, R., Brown, A. H. D., Balmas, V., Saba, E., Schaefer, W., and Attene, G. 2005. Isolation and characterization of the mating-type locus of the barley pathogen Pyrenophora teres and frequencies of mating-type idiomorphs within and among fungal populations collected from barley landraces. Genome 48:855-869.

35. Raymond, M., and Rousset, F. 1995. GENEPOP (version 1.2): Population genetics software for exact tests and ecumenisms. J. Heredity 86:248-249.

36. Robinson, J., and Jalli, M. 1996. Diversity among Finnish net blotch isolates and resistance in barley. Euphytica 92:81-87.

37. Sato, K., and Takeda, K. 1993. Pathogenic variation of Pyrenophora teres isolates collected from Japanese and Canadian spring barley. Bull. Res. Inst. Bioresour. Okayama Univ. 1:147-158.

38. Serenius, M., Manninen, O., Wallwork, H., and Williams, K. 2007. Genetic differentiation in Pyrenophora teres populations measured with AFLP markers. Mycol. Res. 111:213-223.

39. Serenius, M., Mironenko, N., and Manninen, O. 2005. Genetic variation, occurrence of mating types and different forms of Pyrenophora teres causing net blotch of barley in Finland. Mycol. Res. 109:809-817.

40. Smedegård-Petersen, V. 1971. Pyrenophora teres f. maculata f. nov. and Pyrenophora teres f. teres on barley in Denmark. K. Vet. Landboehojsk. Arsskr. 124-144.

41. Steffenson, B., Pederson, J., and Pederson, V. 1999. Common barley diseases in North Dakota. N. D. State Univ. Ext. Serv. Ext. Bull. PP894.

42. Steffenson, B. J., and Webster, R. K. 1992. Pathotype diversity of Pyrenophora teres f. teres on barley. Phytopathology 82:170-177.

43. Tekauz, A. 1985. A numerical scale to classify reaction of barley to Pyrenophora teres. Can. J. Plant Pathol. 7:181-183.

44. Tekauz, A. 1990. Characterization and distribution of pathogenic variation in Pyrenophora teres f. teres and P. teres f. maculata from western Canada. Can. J. Plant Pathol. 12:141-148.

45. Tekauz, A., and Mills, J. T. 1974. New types of virulence in Pyrenophora teres in Canada. Can. J. Plant Sci. 54:731-734.

46. Therrien, M. C., and Wolfe, R. I. 1985. Heartland barley. Can. J. Plant Sci. 65:445-446.

47. Weiland, J. J., Steffenson, B. J., Cartwright, R. D., and Webster, R. K. 1999. Identification of molecular genetic markers in Pyrenophora teres $\mathrm{f}$. teres associated with low virulence on 'Harbin' barley. Phytopathology 89:176-181.

48. Weir, B. C., and Cockerham, C. C. 1984. Estimating F-statistics for the analysis of population structure. Evolution 38:1358-1370.

49. Wilcoxson, R. D., Rasmusson, D. C., and Miles, M. R. 1990. Development of barley resistant to spot blotch and genetics of resistance. Plant Dis. 74:207-210.

50. Wu, H.-L., Steffenson, B. J., and Zhong, S. 2003. Genetic variation for virulence and RFLP markers in Pyrenophora teres. Can. J. Plant Pathol. 25:82-90.

51. Yeh, F. C., Yang, R. C., Boyle, T., Ye, Z., and Xiyan, J. M. 1997. POPGENE, the user-friendly shareware for population genetic analysis. Molecular Biology and Biotechnology Center, University of Alberta, Canada.

52. Zeller, K. A., Bowden, R. L., and Leslie, J. F. 2003. Diversity of epidemic populations of Gibberella zeae from small quadrats in Kansas and North Dakota. Phytopathology 93:874-880.

53. Zhong, S., Leng, Y., Friesen, T. L., Faris, J. D., and Szabo, L. J. 2009. Development and characterization of expressed sequence tag-derived microsatellite markers for the wheat stem rust fungus Puccinia graminis $\mathrm{f}$. sp. tritici. Phytopathology 99:282-289. 